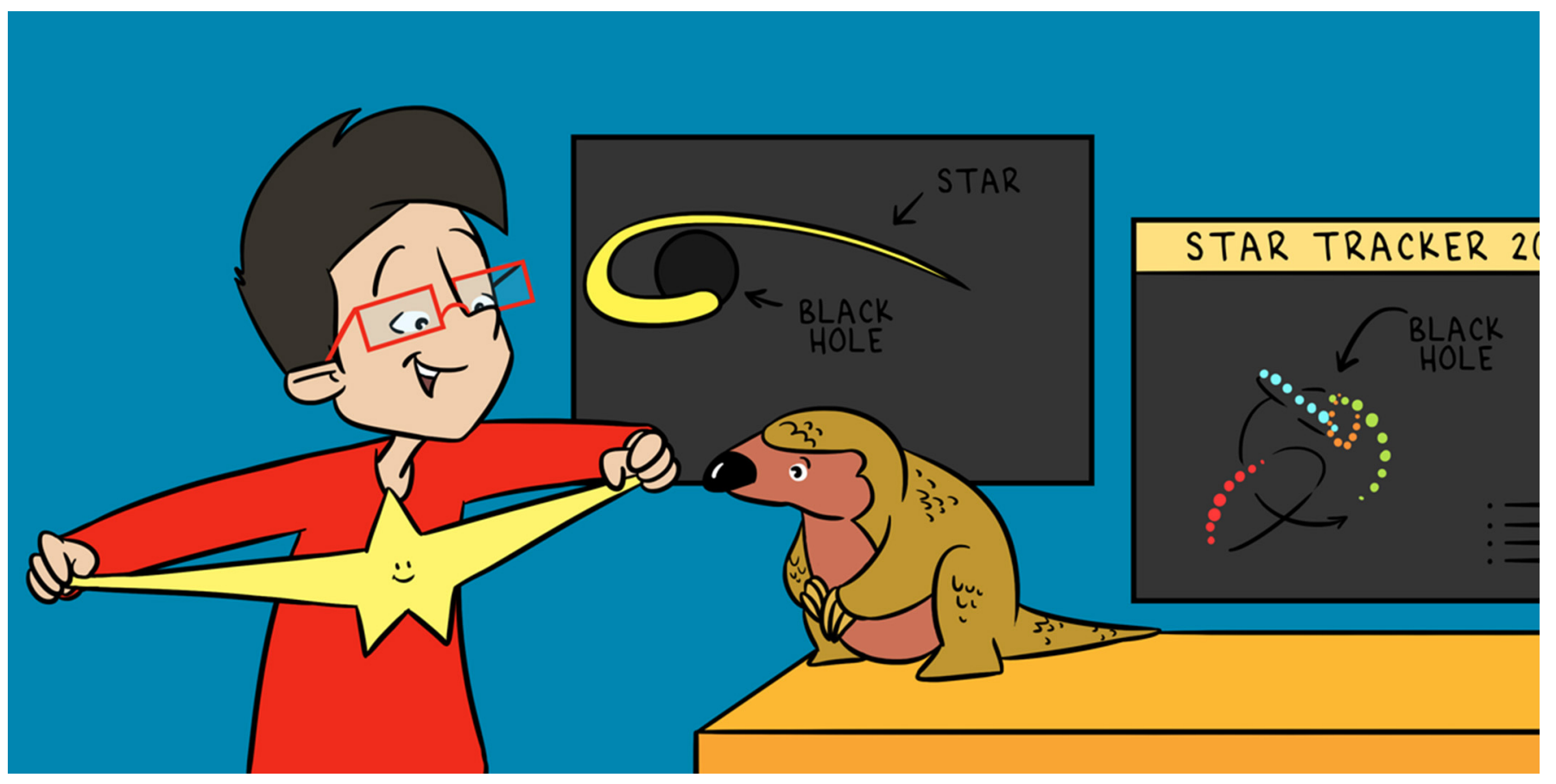

\title{
HOW A STAR"S DEATH CAN REVEAL A BLACK HOLE
}

\section{lair Arcavi *}

The School of Physics and Astronomy, Tel Aviv University, Tel Aviv, Israel

YOUNG REVIEWERS:

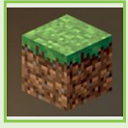

\section{RYAN}

AGE: 15

6. WILLOW

AGE: 13

\section{LIGHT YEAR}

The distance light

travels in a year, which is $5,878,625,370,000$ miles.

1 https://www.nobelprize .org/prizes/physics/ 2020/summary/
Studying invisible objects in space that are hundreds of millions of light years away may sound impossible. But, in recent years, astronomers have developed a new way to investigate a type of invisible and distant objects-super-massive black holes. Black holes are the most densely packed objects in the Universe. When stars get close to super-massive black holes they can be torn apart, which produces a relatively brief but informative flash of light. These star-destroying events can help us to discover the locations of the most massive black holes in the Universe, but only if we know how to find and interpret them. In this article, we will discuss different ways we can "see" black holes, and particularly what we do and do not yet understand about stars getting "tidally disrupted" by them.

\section{SUPER-MASSIVE BLACK HOLES}

It is not easy staring into the center of our galaxy, the Milky Way. Not only because it requires piercing through interstellar "dust" that absorbs visible light, but also because there is a monster lurking there. Two scientists, Andrea Ghez and Reinhard Genzel, received the 2020 Nobel Prize in Physics for looking that monster right in the face. ${ }^{1}$ They developed instruments and methods to obtain a clear picture of what 
Figure 1

The motion of stars around the center of the Milky Way Galaxy. The stars are marked by circles, denoting their positions each year between 1995 and 2008. The star names are shown in the legend. The stars can be seen orbiting an invisible object, located at the very center of the image, having a gravitational force of 4.5 million suns (Photo credit: A. Ghez).

\section{BLACK HOLE}

An object so dense that even light cannot escape its gravitational force.

\section{SUPER-MASSIVE BLACK HOLE}

A black hole that is a million or more times more massive than the sun.

2 Due to the time it takes light to travel across the vast distances in the Universe, the farther away we look, the further back in time we are seeing. This allows every telescope to act like a time machine that can see into the Universe's past.

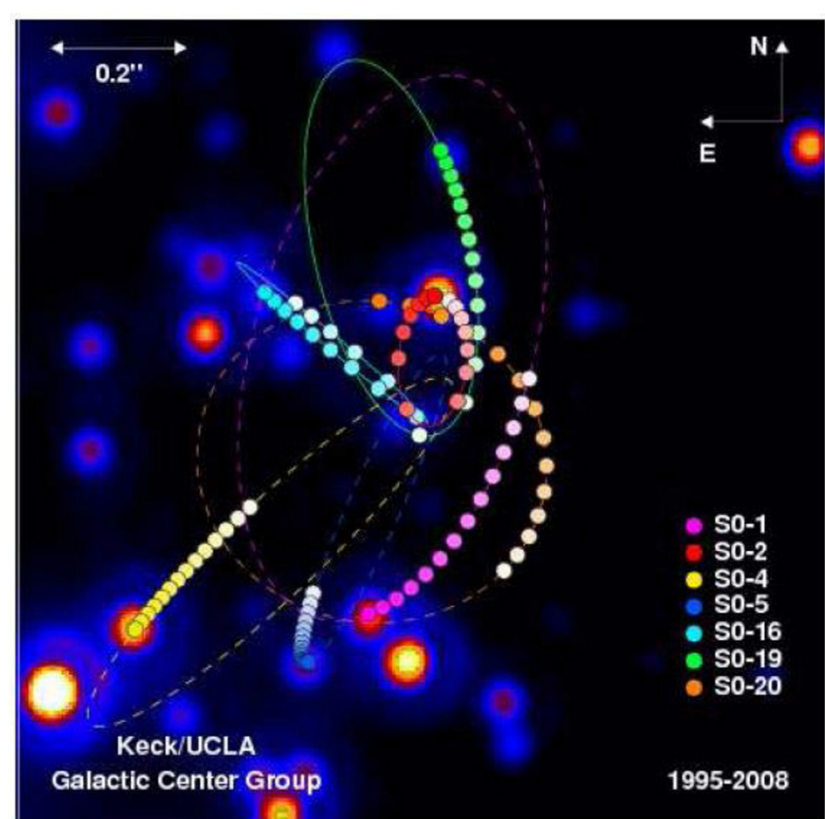

Figure 1

is going on at the heart of the Milky Way. There, they found a group of stars and followed their movements for over a decade. The stars were seen to orbit a point in space as if they were affected by the gravity of 4.5 million suns (Figure 1). But, at that point in space, nothing was emitting any light. What object could produce the gravitational force of 4.5 million suns and not produce any light? A black hole. And not just any black hole, but a super-massive black hole.

Black holes are extremely dense objects, equivalent to shrinking the entire Earth down to the size of a ping-pong ball. The black hole at the center of the Milky Way, with its mass of 4.5 million suns, could fit inside the orbit of Mercury. The Milky Way is not the only galaxy hiding such a monster. In fact, super-massive black holes probably lie at the center of all large galaxies [1] and can be as massive as a 100 billion suns [2]. We do not understand how the universe forms such monstrous objects. Even more puzzling is that super-massive black holes can be seen already in the early Universe-when the Universe was younger than the time it should take such black holes to form [3]. ${ }^{2}$

\section{SEEING THE INVISIBLE}

The challenge in trying to investigate black holes is that they are, obviously, black - they do not emit light-and so they are very hard to detect. The method used to discover the super-massive black hole in the center of our galaxy-measuring the movement of stars around it-can be used only for the closest galaxies. Beyond those, even our 


\section{Figure 2}

The silhouette of the super-massive black hole at the center of a galaxy called M87, seen against the gas that surrounds it, which heats up and shines (Photo credit: Event Horizon Telescope).

3 https://eventhorizon telescope.org/pressrelease-april-10-2019astronomers-capturefirst-image-black-hole

TIDAL DISRUPTION

\section{EVENT (TDE)}

The act of a star being torn apart after getting too close to a black hole.

4 https://scijinks.gov/ tides/

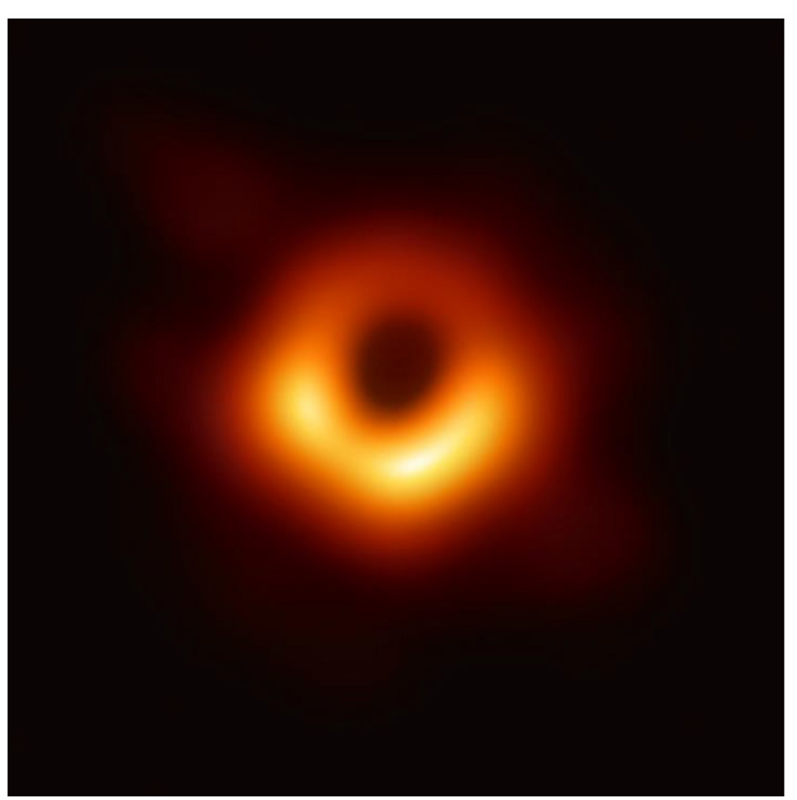

Figure 2

most sophisticated telescopes cannot distinguish the movements of individual stars in the centers of galaxies.

There is another way to "see" black holes from greater distances. About $10 \%$ of super-massive black holes are surrounded by gas, which slowly fall onto them $[4,5]$. Like water down a bathtub drain, as the gas approach the black hole they move faster and faster. The gas around black holes can reach velocities so high that they heat up and shine. The "silhouette" of the super-massive black hole in the center of the galaxy M87 was recently imaged against the background of such light (Figure 2). ${ }^{3}$ In fact, gas that is at the verge of falling onto a super-massive black hole is the brightest source of light in the Universe. With this light, not only can super-massive black holes be identified, but their mass and spin can be calculated.

But what about the other $90 \%$ of super-massive black holes that are not actively swallowing gas? Such black holes are completely invisible to us...unless (and this is a very rare "unless"-once every few hundred thousand years in a galaxy), an unlucky star happens to get close to the super-massive black hole and falls victim to what is called a tidal disruption event (TDE) [6].

\section{TIDAL DISRUPTION EVENTS}

Tides on Earth are caused by the gravitational force of the Moon, which pulls more strongly on the water on the side of the Earth that is facing the Moon than on the water on the opposite side. ${ }^{4}$ Similarly, the unlucky star approaching a black hole will feel a stronger gravitational force acting on the side that is facing the black hole. Gravitational force 
Figure 3

Illustration of a star being torn apart by a black hole (Image credit: NASA/CXC/M. Weiss).

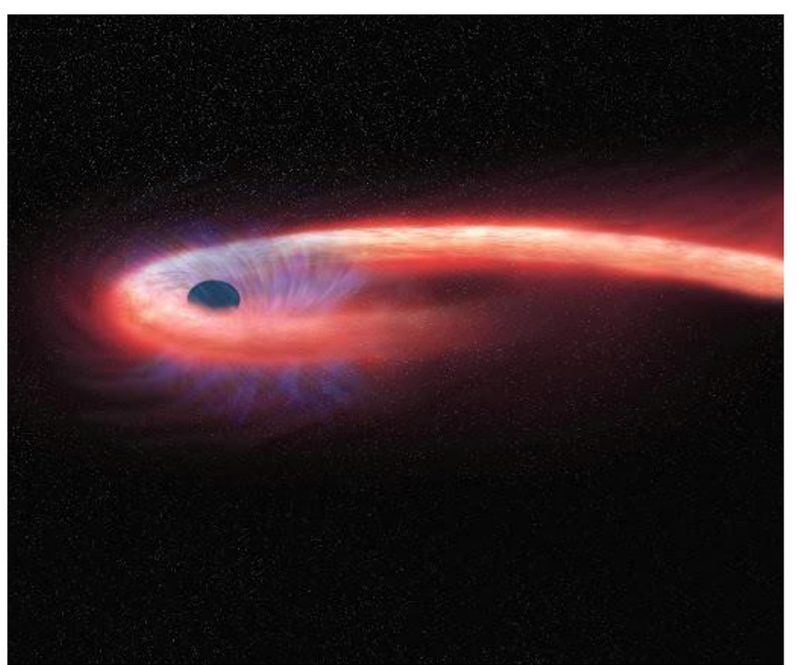

Figure 3

is also what holds all stars together. So, if the star gets close enough to the black hole, the difference in gravitational force acting on each side of the star can be stronger than the gravitational force holding the star together. As a result, the star will be spaghettified-it will be stretched out into a long strand (Figure 3). To understand why this happens, imagine you are falling in, feet first, toward a black hole. Since your feet are closer to the black hole than your head is, they feel a stronger gravitational force and are pulled in faster than your head is. This effect will cause your whole body to stretch like a strand of spaghetti. In the case of a star, it actually gets torn apart-half of it will enter into orbit around the black hole, and half will be ejected away from the black hole, into space.

Like the gas around active black holes, the half-star that stays in orbit around the black hole will orbit closer and closer to it, speed up, heat up, and emit light. However, unlike active black holes that have a huge supply of gas that generates light continuously, in the case of a lone disrupted star, the light will fade within weeks or months, once all the material from the orbiting half-star falls into the black hole. From the black hole's point of view, it is a snack rather than a feast. But for scientists studying black holes, it is an opportunity that reveals the existence and properties of an otherwise invisible object.

\section{THE MYSTERIES OF TIDAL DISRUPTION EVENTS}

The TDE phenomenon was predicted as early as the 1970s [7], but we have only recently found and identified it using telescopes [8]. One of the reasons that it took so long to start finding these events was that the properties of the light emitted by TDEs are different than what we expected. When gas warms up before falling onto a black hole, it emits very high-energy $\mathrm{X}$-ray light. However, the TDEs we are finding 


\section{ULTRAVIOLET}

LIGHT

Light at

shorter wavelengths

than visible blue light today emit mostly visible and ultraviolet light, at temperatures and brightnesses that are 10 to 100 times lower than what was predicted. Why is that? We do not really know.

Another mystery relating to TDEs is the galaxies in which we find them $[9,10]$. Almost all TDEs discovered today are found in galaxies that experienced a burst of star formation a few hundred million years ago. These post-starburst galaxies are rare-they make up only a few percent of the galaxies in the universe-but they host about 9 out of 10 TDEs. How does a burst of star formation relate to an increased TDE rate a few hundred million years later? We do not fully understand that either.

We set out to find TDEs around super-massive black holes so that we could identify and study the black holes themselves. We indeed found the TDEs we were looking for but, as happens often in science, they presented us with new and unexpected puzzles. As we discover and study more TDEs, we hope that we can both solve their puzzles and learn to use them as tools for investigating the monsters that lie at the heart of every galaxy.

\section{REFERENCES}

1. Kormendy, J., and Richstone, D. 1995. Inward bound-the search for supermassive black holes in galactic nuclei. Ann. Rev. Astron. Astrophys. 33:581-624. doi: 10.1146/annurev.aa.33.090195.003053

2. Kozłowski, S. 2017. Virial black hole mass estimates for 280,000 AGNs from the SDSS broadband photometry and single-epoch spectra. Astrophys. J. Suppl. Ser. 228:9. doi: 10.3847/1538-4365/228/1/9

3. Bañados, E., Venemans, B., Mazzucchelli, C., Farina, E. P., Walterm F., Wang, F., et al. 2018. An 800-million-solar-mass black hole in a significantly neutral Universe at a redshift of 7.5. Nature. 553:473-6. doi: 10.1038/nature25180

4. Antonucci, R. 1993. Unified models for active galactic nuclei and quasars. Ann. Rev. Astron. Astrophys. 31:473-521. doi: 10.1146/annurev.aa.31.090193. 002353

5. Shankar, F. 2009. The demography of supermassive black holes: growing monsters at the heart of galaxies. New Astron. Rev. 53:57-77. doi: 10.1016/j.newar.2009.07.006

6. Rees, M. J. 1988. Tidal disruption of stars by black holes of $10^{6}-10^{8}$ solar masses in nearby galaxies. Nature. 333:23-8. doi: 10.1038/333523a0

7. Hills, J. G. 1975. Possible power source of Seyfert galaxies and QSOsrt. Nature. 254:295-8. doi: 10.1038/254295a0

8. van Velzen, S., Holoien, T. W. S., Onori, F., Hung, T., and Arcavi, I. 2020. Optical-ultraviolet tidal disruption events. Space Sci. Rev. 216:124. doi: 10.1007/s11214-020-00753-z

9. Arcavi, I., Gal-Yam, A., Sullivan, M., Pan, Y. C., Bradley Cenko, S., Horesh, A, et al. a continuum of $\mathrm{H}$ - to He-rich tidal disruption candidates with a preference for E+A galaxies. Astrophys. J. 793:38. Available online at: https://iopscience. iop.org/article/10.1088/0004-637X/793/1/38 
10. French, K. D., Wevers, T., Law-Smith, J., Graur, O., and Zabludoff, A. I. 2020. the host galaxies of tidal disruption events. Space Sci. Rev. 216:32. doi: 10.1007/s11214-020-00657-y

SUBMITTED: 19 May 2021; ACCEPTED: 13 December 2021; PUBLISHED ONLINE: 10 January 2022.

EDITED BY: Edward Gomez, Las Cumbres Observatory Global Telescope Network, United States

CITATION: Arcavi I (2022) How a Star's Death Can Reveal a Black Hole. Front. Young Minds 9:712082. doi: 10.3389/frym.2021.712082

CONFLICT OF INTEREST: The author declares that the research was conducted in the absence of any commercial or financial relationships that could be construed as a potential conflict of interest.

COPYRIGHT () 2022 Arcavi. This is an open-access article distributed under the terms of the Creative Commons Attribution License (CC BY). The use, distribution or reproduction in other forums is permitted, provided the original author(s) and the copyright owner(s) are credited and that the original publication in this journal is cited, in accordance with accepted academic practice. No use, distribution or reproduction is permitted which does not comply with these terms.

\section{YOUNG REVIEWERS}

\section{RYAN, AGE: 15}

I really enjoy coding and I love Rubik's cubes. I also really love playing Minecraft.

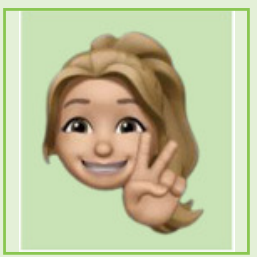

\section{WILLOW, AGE: 13}

My name is Willow (named after the character from Buffy the vampire slayer), I am an 8th grader at a local middle school. I love playing soccer and kicking around a ball with my dog, Suki. In my free time, I enjoy baking and hanging out with friends.

\section{AUTHOR}

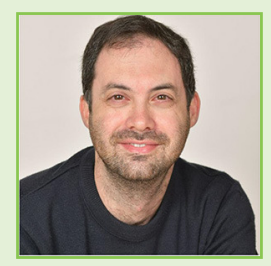

\section{IAIR ARCAVI}

lair (pronounced "ya-eer") Arcavi is an astrophysicist at Tel Aviv University. He studies fleeting energetic events in the Universe, like exploding stars (supernovae), stars torn apart by black holes (tidal disruption events) and merging neutron stars. In order to catch and observe these events he uses a network of robotic telescopes spread out around the world, that can react within minutes. When not doing astronomy, lair enjoys photography, traveling to new places, and eating good chocolate. *arcavi@gmail.com 